application front-end was developed in Ionic. The back-end consists of an internal SQLite Database that provides the mobile app with direct read and write access to the data, stored locally in a single file.

The mobile app developed allows patients to record important information about their condition. The app will support patient self-management, and empower patients when accessing services outside of their normal care team. This app could be modified to support a variety of childhood chronic conditions.

\section{DIGITALISATION OF MYEYES, A VISION RELATED PATIENT REPORTED OUTCOME MEASURE}

${ }^{1}$ Minu Choi, ${ }^{1}$ Mr Simon Kanani, ${ }^{1}$ Mr James Malkin, ${ }^{2}$ Ms Daiana Bassi, ${ }^{2}$ Mrs Sue Conner, ${ }^{1}$ Yun Fu, ${ }^{1}$ Dean Mohamedally, ${ }^{2}$ Gemma Molyneux, ${ }^{1}$ Graham Roberts, ${ }^{2}$ Neil J Sebire, ${ }^{3}$ Alexandra Robertson, ${ }^{3}$ Jugnoo Rahi, ${ }^{3}$ Ameenat Lola Solebo. ' UCL Department of Computer Sciences; ${ }^{2}$ DRIVE, Great Ormond Street Hospital for Children NHS Foundation Trust; ${ }^{3}$ UCL Great Ormond Street Institute of Child Health

10.1136/archdischild-2020-gosh.22

Childhood visual impairment (VI) has significant impact on the child and with far-reaching consequences for the child's social and educational experiences and future career prospects.

The VQoL_CYP and FVQ_CYP are validated patientreported outcome measures (PROMs) which capture visionrelated quality of life (VQoL) and functional vision (FV) and are designed for use by children and young people living with VI, to capture children's own perspectives of the impact of VI. They are currently used as paper based questionnaires in research and routine clinic practice. However, clinicians and families would benefit from digitalising the process, allowing patients to complete questionnaires electronically with data automatically collected into a database for analysis.

In collaboration with UCL computer science, through the industry exchange network, researchers at GOSH and UCL GOS Institute of Child Health developed a proof of concept app was created to deploy both instruments digitally (collectively, 'MyEyes'), complete with a database for analysis. The web application was developed using Django with a PostgresSQL database. The mobile app was developed using Ionic.

The digitalisation of the questionnaires has the potential to improve the child's experience of self-completion. Deployment via a mobile app allows patients to complete questionnaires at sequential time-points, in-between normal clinic visits, thus providing clinicians with more information about their eye condition over time.

To gain feedback on the technology, patients and families will now test the prototype mobile app. This project demonstrates how PROMs can be digitalised, supporting a more efficient collection process for important patient-generated data. This prototype could serve as an example of how other PROMs in use at the hospital could be deployed digitally.

\section{RAPID DESIGN AND DEPLOYMENT OF AN ADULT CRITICAL CARE TRANSPORT TEAM - THE LAUNCH OF BIG CATS}

Cathy Roberts, Mark Clement. Children's Acute Transport Service (CATS)

10.1136/archdischild-2020-gosh.23
The Children's Acute Transport service (CATS) is a standalone paediatric critical care transport service in the north thames region. In April 2020, demand for adult intensive care beds exceeded surge capacity in some North Central London (NCL), units which led to an urgent need to transfer COVID$19+$ ve adults between units.

A newly formed adult critical care transport service in the NCL region became overwhelmed with requests and CATS were asked to assist. Big CATS was launched within 48 hours, activated 16 times and offered a 7 day a week daytime service without denuding paediatric capacity.

\section{MONITORING BONE HEALTH IN CHILDREN AND YOUNG PEOPLE WITH COELIAC DISEASE - A CLINICAL AUDIT}

Sarah Khweir, Fevronia Kiparissi, Edward Gaynor. GOSH

\subsection{6/archdischild-2020-gosh.24}

Introduction Monitoring of bone health via bone density in coeliac disease (CD) using dual-energy X-ray absorptiometry (DEXA) in childhood is recognised in clinical practice, however there is variance in frequency of monitoring. At Great Ormond Street Hospital (GOSH) the coeliac protocol is to perform a DEXA after diagnosis. National guidelines are nonspecific about the paediatric population. This audit explored whether children with $\mathrm{CD}$ are meeting current guidance of DEXA scan monitoring at GOSH and whether those who have had DEXA scans had a change in their management.

Methods Retrospective clinical audit, registered with the research and development team. Data collected via electronic patient records (EPR) April 2020 on all patients seen in the dietetic led coeliac clinic.

Results 31 patients in dietetic led coeliac clinic. Median age at diagnosis was three years. $87 \%$ patients had a DEXA scan, of which $81 \%$ of were normal. Scans were performed a median 3.5 years after diagnosis. $2 / 5$ patients with abnormal DEXA had tTGA's of $8-12.5 \mathrm{U} / \mathrm{ml}$. BMAD $\mathrm{z}$-scores were completed in $78 \%$ of patients with mean -0.27 . Serum levels of vitamin D were $>25 \mathrm{nnmol} / \mathrm{L}$ in $100 \%$ patients but $45 \mathrm{nmol}-60 \mathrm{nmol} / \mathrm{L}$ in children with abnormal DEXA. No child had faltering growth at time of DEXA.

Conclusions As children are being diagnosed early, and there is a reversal in low bone mineral density on a gluten free diet, there are fewer indications to measure DEXA routinely. DEXA should therefore be only reserved for 'at risk' groups. Vitamin D supplementation of 10 ug daily and annual monitoring should be routinely advised.

\section{IDENTIFYING EFFECTIVE WAYS TO COMMUNICATE HEALTHCARE SCIENCE CAREERS TO SECONDARY SCHOOL STUDENTS}

${ }^{1}$ Sarah Radi, ${ }^{2}$ Victoria Heath. 'Secondary school student on work experience placement; ${ }^{2} \mathrm{GOSH}$

\subsection{6/archdischild-2020-gosh.25}

Engaging with secondary school students to raise awareness of the careers available in healthcare science is a vital step in recruitment and development of the future workforce. In order for healthcare science careers events to be successful, it 